\title{
Psoriasis and smoking: links and risks
}

This article was published in the following Dove Press journal:

Psoriasis: Targets and Therapy

27 May 2016

Number of times this article has been viewed

\author{
Luigi Naldi',2 \\ 'Department of Dermatology, Azienda \\ Ospedaliera Papa Giovanni XXIII, \\ Bergamo, Italy; ${ }^{2}$ Study Centre of \\ the Italian Group for Epidemiologic \\ Research in Dermatology (GISED), \\ Bergamo, Italy
}

Abstract: Smoking is a complex environmental exposure influenced by genetic, environmental, and social factors. Nicotine is the principal alkaloid in tobacco that mediates the addicting effects of tobacco products. Tobacco is a mixture of more than 7,000 chemicals, and smoking is recognized as a risk factor for many diseases in humans, including cardiovascular and pulmonary disease and several cancers, and is the single most preventable cause of mortality worldwide. A number of inflammatory immune-related conditions have been associated with smoking, including psoriasis. Smoking affects the onset of psoriasis. In a pooled analysis of 25 case-control studies, the odds ratio of psoriasis among smokers was 1.78 (95\% confidence interval [CI]: 1.53-2.06). A dose-effect relationship is also documented. In a pooled analysis of three cohort studies, the risk of incident psoriasis was 1.81 (95\% CI: 1.38-2.36) in those who smoked 1-14 cigarettes per day, and 2.29 (95\% CI: 1.74-3.01) in those who smoked $\geq 25$ cigarettes per day. Smoking also impacts on the clinical severity of psoriasis, its response to treatment, and explains some of the associated comorbidities, eg, cardiovascular disease, inflammatory bowel disease, and several cancers (especially those of the respiratory tract). Data on the role of smoking in psoriatic arthritis are less consistent compared with those concerning psoriasis. Several pathophysiological mechanisms may explain the association of psoriasis with smoking, including oxidative stress, interaction with signaling pathways active in psoriasis, and vascular influences. In conclusion, psoriasis is just one of the many diseases associated with smoking, but it is visible and disabling. Dermatologists could play a major role in reducing the health burden of smoking by influencing the patients to change their behavior.

Keywords: smoking, nicotine dependence, risk, psoriasis, onset, prognosis, comorbidities

\section{Smoking: a complex environmental exposure and a threat to health}

Smoking is the most common way of consuming tobacco, and tobacco is the substance most commonly smoked. Tobacco contains the alkaloid nicotine, which is a stimulant that can lead to dependence. It has been estimated that about one out of three people who have ever attempted to smoke become usual smokers. ${ }^{1}$

Smoking is a complex environmental exposure influenced by behavioral models and values. Smoking tobacco among natives in Americas had been a social and ceremonial activity. Tobacco was imported to Europe in the late 16th century, and tobacco smoking became increasing popular after the development of the automated cigarette-rolling apparatus at the end of the 19th century. In the early 1960, it reached its peak, and the rates of tobacco consumption started declining thereafter in the developed countries
Department of Dermatology, Azienda Ospedaliera Papa Giovanni XXIII, Piazza OMS I, 24100 Bergamo, Italy

Tel +39035 2278719 ext 720

Fax +390352278 673

Email luigi.naldi@gised.it
Psoriasis: Targets and Therapy 20|6:6 65-7|

65

Dovepress for in


as a consequence of educational campaigns and restriction on smoking in public places. However, the rates continue to rise in developing countries. ${ }^{2}$

Cigarette smoking is recognized as a risk factor for several diseases in humans, and it remains the single most important source of preventable global mortality. The association of smoking with major cardiovascular disease, chronic obstructive lung disease, peptic ulcer, and several cancers (particularly those of the respiratory tract) is well established. ${ }^{3-5}$ In addition, smoking has been linked with a number of chronic immune-mediated inflammatory diseases, including psoriasis. ${ }^{6}$

Tobacco smokers are exposed to a mixture of over 7,000 chemicals, distributed in a particulate and a vapor phase (Table 1). ${ }^{7}$ The composition may vary with the cigarette model and brand.

Nicotine is the main alkaloid in tobacco, and it is responsible for the addicting effects of tobacco smoking. Nicotine is quickly absorbed not only through the alveolar spaces in the lung but also through the skin and the intestinal mucosa. It is metabolized mainly by the liver to several metabolites, some of which maintain pharmacological activity. ${ }^{8,9}$ Nicotine acts on various subtypes of nicotinic acetylcholine receptors. They are found not only in the nervous system and adrenal

Table I Selected components in tobacco smoke

\begin{tabular}{l} 
Particulate phase \\
\hline Polycyclic aromatic hydrocarbons \\
N-heterocyclic amines \\
Nicotine \\
Phenol, cresol \\
Hydroquinone \\
Naphtylamine \\
Benzo(a)pyrene \\
Indole, carbazole \\
Trace metals (eg, arsenic) \\
\hline Vapor phase \\
\hline Nitrogen \\
Oxygen \\
Carbon dioxide \\
Carbon monoxide \\
Formaldehyde \\
Hydrocyanic acid \\
Acetaldehyde \\
Acrolein \\
Ammonia \\
Nitrogen oxides \\
Nitrosamines \\
Hydrazine \\
Vinyl chloride \\
Not
\end{tabular}

Note: The particulate and vapor phases are operationally defined as fractions of cigarette smoke that are retained or passed through a Cambridge filter, respectively. medulla, but have also been identified in other tissues, such as skin keratinocytes and inflammatory cells, eg, monocytes and dendritic cells. The interaction between nicotine and nicotinic acetylcholine receptors results in an initial temporary activation of ganglionic transmission, followed by a lasting depression (biphasic effect), and also results in the release of catecholamines from the adrenal medulla and postganglionic sympathetic neurons. The role of nicotine in the skin and inflammatory cells is less clearly delineated. It might facilitate keratinocyte adhesion and upward migration in the epidermis, and may have an immunomodulatory effect. ${ }^{10}$

Among other substances, 1,3-butadiene carries the highest risk of cancer; acrolein and acetaldehyde are main respiratory irritants; and arsenic, cyanide, and cresols are leading sources of cardiovascular risk. Inflammatory processes can also be affected by smoking. Chronic exposure to benzo(a) pyrene induces dose-related decreases in the mass of lymphoid tissue, and the unsaturated aldehyde acrolein appears to affect the neutrophil function. ${ }^{11}$ The effects of smoking can be modulated by sex. A meta-analysis documented that females have a higher risk compared to males of overall morbidity and mortality associated with smoking at any level of exposure. ${ }^{12}$

After the recognition of the adverse effects of smoking in the early $1960 \mathrm{~s}$, the tobacco producers started developing cigarettes with low tar and nicotine machine-measured yields. ${ }^{13}$ These changes over the last six decades did not have an impact on the overall morbidity and mortality from smoking and may have even interfered with attempts to quit smoking by the public and with preventive campaigns. ${ }^{14}$

Electronic cigarettes (e-cigarettes) are battery-powered vaporizers that simulate the feeling of smoking, but without burning tobacco. Their use is commonly called "vaping". E-cigarette liquids are typically solutions of glycerol and/or propylene glycol, nicotine, and flavors. The benefits and the health risks of e-cigarettes are uncertain. ${ }^{15,16}$ They can help people quit smoking, but they have not been proven better than regulated medication. It has been shown that hemiacetals containing formaldehyde can be formed during the "vaping" process, which may carry risk of cancer. These results are still debated. ${ }^{17}$

Tobacco dependence is influenced by a variety of environmental and genetic factors. The same factors may, in turn, play some causal role in smoking-related diseases. ${ }^{18}$ The risk of becoming a smoker has been associated with a lower socioeconomic status, with peer smoking, and even with maternal smoking during pregnancy. ${ }^{19}$ A substantial hereditary component has been associated with selected features of smoking habits, such as age at initiation and intensity of smoking, with 
heritability estimates in twin studies, ranging from $<0.3$ to $>0.8 .^{20,21} \mathrm{~A}$ meta-analysis has suggested that heritability of smoking habits may vary with sex, with a higher heritability component for smoking initiation in females and a higher heritability component for persistence in males. ${ }^{22}$ Smoking persistence and the ability to stop smoking may have an even stronger genetic component. Molecular studies have been conducted to assess genetic traits associated with smoking habits. Candidate gene studies have particularly focused on genetic variations in neurotransmitter pathways and nicotine-metabolizing enzymes. The candidate genes with the strongest evidence of an association with smoking behavior are $C Y P 2 A 6,5 H T T$ and single-nucleotide polymorphisms in the $C H R N A 5 / A 3 / B 4$ gene cluster. ${ }^{23}$

\section{Cigarette smoking as a risk factor for the onset of psoriasis}

Psoriasis is a chronic inflammatory skin condition that affects $\sim 3 \%$ of the general population. It is clinically heterogeneous, with distinct, partly overlapping phenotypes, including chronic plaque, guttate, erythrodermic, and pustular varieties. Incident psoriasis has been linked with smoking habits in both case-control and cohort studies (Figure 1). Ideally, assessment of risks should be done separately for each clinical variant. This has been rarely done.

In a meta-analysis, ${ }^{24}$ a total of 25 case-control studies and four cohort studies were analyzed. In case-control studies, pooled odds ratio (OR) of psoriasis among smokers was 1.78
(95\% confidence interval [CI]: 1.53-2.06). Metaregression did not reveal any significant sources of heterogeneity. The analysis of the association between psoriasis and a history of former smoking, including seven studies, found a pooled OR of 1.62 (95\% CI: 1.33-1.98) for psoriasis in former smokers. ${ }^{24}$ Few studies provided data on duration and intensity of smoking in relation to psoriasis.

As for cohort studies, a pooled analysis of data from three studies, namely, the Nurses' Health Study (NHS), the Nurses' Health Study II (NHS II), and the Health Professionals' Follow-Up Study, gave a pooled OR for psoriasis of 1.94 (95\% CI: 1.64-2.28). Data from the General Practice Research Database study provided similar results with OR for psoriasis in current smokers of 1.45 (95\% CI: 1.31-1.59). Pooled analysis of the three aforementioned cohort studies also gave evidence of an association of the risk of psoriasis with smoking intensity and duration. The risk of incident psoriasis was 1.81 (95\% CI: 1.38-2.36) in those who smoked 1-14 cigarettes per day and 2.29 (95\% CI: 1.74-3.01) in those who smoked $\geq 25$ cigarettes per day. While the risk of psoriasis in subjects with smoking duration $<10$ years was almost the same as nonsmokers, those with a smoking duration $\geq 30$ years had nearly twice the risk of nonsmokers (adjusted OR: 1.99, 95\% CI: 1.75-2.25). The population-attributable risk of smoking for incident psoriasis was $15 \%$ in the NHS II cohort and $20 \%$ in the NHS cohort. ${ }^{24}$ A case-control study provided data on the risk of incident psoriasis according to sex. It showed variations between the sexes, with an OR for

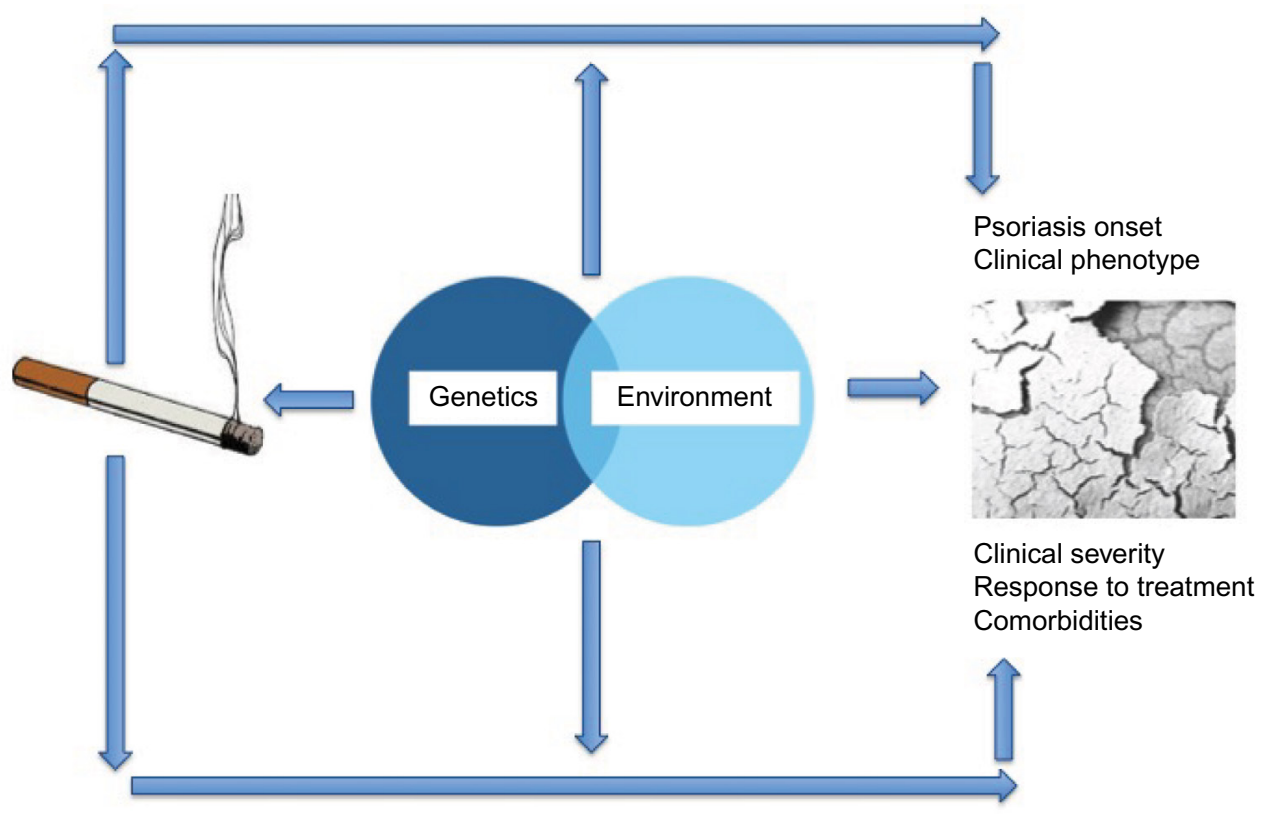

Figure I Schematic representation of the relationship between smoking and psoriasis. 
smoking 15 cigarettes or more of 3.2 in women and 1.6 in men. In the study, the OR for pustular lesions in smokers was 9.9. ${ }^{25}$ Tobacco smoking has been strongly associated with palmoplantar pustulosis, a condition that is now considered separate from psoriasis. ${ }^{26}$

Recent data, mainly from Chinese investigators, point to a possible interaction between smoking and genes involved in psoriasis susceptibility or smoking habits. ${ }^{27-30} \mathrm{~A}$ case-control study showed that the risk of psoriasis for the combination of being a smoker and carrying the aplotype HLA-Cw6 was approximately 11 -fold higher compared with nonsmokers without HLA-Cw6. ${ }^{27}$ Another study showed interaction between single-nucleotide polymorphisms at locus CSMD1, a tumor-suppressor gene expressed in areas of regenerative growth, and cigarette smoking. ${ }^{28}$ Another study found an interaction between smoking and the gene for CYP1A1, a member of the cytochrome P450 family involved in the metabolism of polycyclic aromatic hydrocarbons produced during tobacco combustion. ${ }^{30}$

Several pathophysiological mechanisms may explain the association of smoking with psoriasis. ${ }^{31}$ Smoking causes oxidative stress and production of dangerous free radicals, interfering with signal pathways relevant in psoriasis such as mitogen-activated protein kinase, nuclear factor kappa B (NF- $\mathrm{KB}$ ), and JAK-STAT pathways. Nicotine also induces an increased secretion of several cytokines such as interleukin (IL)-12, tumor necrosis factor (TNF), IL-2, and granulocytemonocyte colony-stimulating factor, which play a crucial role in the pathogenesis of psoriasis. ${ }^{32}$ In vitro studies in human $\mathrm{HaCaT}$ keratinocytes have shown that the increased expression and secretion of TNF due to cigarette smoke extract is mediated by the increased expression, promoter activity and nuclear translocation of the Egr-1 transcription factor, a protein, and a transcription factor encoded by the EGRI gene. ${ }^{33}$ It has also been documented that the expression of vascular endothelial growth factor, an important element in angiogenesis, can be modified by smoking. ${ }^{34}$

\section{The role of smoking in comorbidities and prognosis of psoriasis}

Not only has smoking been associated with the onset of psoriasis but it has also been linked with the severity of the disease and response to treatment. An Italian cross-sectional study documented that patients smoking $>20$ cigarettes daily had a twofold increased risk of presenting with severe psoriasis than smokers of less than ten cigarettes daily. ${ }^{35} \mathrm{In}$ a study of male psoriatic patients, smoking more than ten cigarettes per day was significantly associated with increased severity especially on the extremities. ${ }^{36}$ Similarly, another study found a more severe skin involvement in smokers than in nonsmokers and also a reduced likelihood of experiencing periods of disease remission. ${ }^{37}$ Negative results also exist. A retrospective study of 66 psoriatic patients did not show any significant difference between smokers and nonsmokers in Physician Global Assessment after 3-16 months of systemic treatment, nor in the average number of systemic treatments tried per patient. ${ }^{38}$ It should be noted that the study was underpowered to see even a large-to-moderate effect of smoking.

A recent systematic review of adherence to treatment in patients with psoriasis identified three studies assessing the role of smoking for adherence. ${ }^{39}$ Two studies reported adherence to be greater for nonsmokers compared with smokers, while another study reported no association. Larger prospective studies are warranted in this area. Interestingly, in a series of 110 psoriatic patients treated with anti-TNF, smoking together with increased body mass index and a high baseline psoriasis area and severity index (PASI) score was a risk factor for a lack of response to the treatment. ${ }^{40}$

A few data on genetic-environmental interaction exist for the relationship between severity of psoriasis and smoking. A hospital-based case-control study of 672 subjects showed a combined effect of rs6474412 genotype (TT) and smoking behavior on disease severity (OR for severe disease: 5.95; 95\% CI: 1.39-25.31). ${ }^{41}$

Many of the comorbidities associated with psoriasis can be related, at least partly, with smoking. They include cardiovascular disease, inflammatory bowel disease (IBD), and several cancers (especially those of the respiratory tract). ${ }^{42,43}$

Data on the role of smoking in psoriatic arthritis are less consistent compared with those concerning psoriasis. Sparse evidence regarding the association between smoking and psoriatic arthritis has been published. One study showed that smoking may accelerate the onset of arthritis in patients with psoriasis, while it may delay the onset of arthritis in healthy participants. ${ }^{44}$ Another case-control study did not find an increased risk of psoriatic arthritis among smokers with psoriasis. ${ }^{45}$ A larger cohort study based on the NHS II (1991-2005) documented that smoking was associated with an elevated risk of incident psoriatic arthritis, with a relative risk of $1.54(95 \%$ CI: 1.06-2.24) for past smokers and 3.13 (95\% CI: 2.08-4.71) for current smokers. With increasing smoking duration or number of cigarettes smoked, the risk of psoriatic arthritis increased monotonically. The increase in risk was particularly significant for patients with more severe phenotypes. ${ }^{46}$

Smoking can affect disease activity in psoriatic arthritis. An analysis of data from the Bath longitudinal cohort 
involving 267 patients with psoriatic arthritis and disease duration $\geq 10$ years showed that smoking, delay of diagnosis, older age at diagnosis, and female sex were all associated with worse physical function. ${ }^{47}$ Data from the Danish DANBIO registry, including 1,148 patients with psoriatic arthritis and known smoking status, documented that smokers had worse baseline patient-reported outcomes, shorter treatment adherence, and poorer response to TNF compared to nonsmokers, with a more pronounced effect in men and in patients treated with infliximab or etanercept. ${ }^{48}$

Recent epidemiological studies showed that periodontitis could represent an independent risk factor for incident psoriasis. ${ }^{49}$ The pathogenesis of psoriasis and periodontal disease is linked with common immune-related pathways. In a hospital-based case-control study enrolling 82 psoriatic patients and 89 controls, periodontal disease was linked with psoriasis (OR: 4.37 [95\% CI: 1.11-17.16]), and the risk of severe periodontal disease among psoriatic patients was six times higher in smokers than that in nonsmokers. ${ }^{50}$

Smoking has been linked with the risk of severe infections in patients treated with systemic agents for psoriasis. In a cohort study of systemically treated patients, the hazard ratio (HR) of severe infections in smokers was $1.43(95 \%$ CI: 1.08-1.88) compared with nonsmokers. ${ }^{51}$

Psoriasis is an emerging paradoxical side effect of antiTNF treatment in patients with IBD. Several studies have pointed to smoking as a risk factor for such a paradoxical reaction. A recent retrospective cohort study of 402 patients with IBD who started anti-TNF- $\alpha$ at a single center showed that smoking was an independent risk factor for new onset psoriasis, with an HR of 2.37 (95\% CI: 1.36-4.48); on the contrary, concomitant immunosuppressive therapy was a protective factor, with an HR of 0.33 (95\% CI: 0.12-0.92). ${ }^{52}$

\section{Reducing the health burden of smoking: the role of dermatologists}

About one-half of people with a long-term history of smoking, especially those who started smoking in adolescence, will ultimately die from the health consequences of tobacco use. One-half of these deaths will occur in middle-aged people, with a reduction of 20-25 years of normal life expectancy. ${ }^{53}$ Nicotine dependence is the main reason why individuals persist smoking.

Psoriasis is just one of the many diseases associated with smoking. Nonetheless, it is a visible and debilitating condition, and patients may be more motivated to consider quitting smoking because of it than in consideration of other nonvisible health problems. Dermatologists could play a major role in inducing their patients to stop smoking. All psoriatic patients should be asked if they use tobacco and should have their smoking status documented. An initial prompt from a health professional is important to stimulate awareness of risks. ${ }^{54}$ Referral to intensive support programs should be offered, such as the NHS Stop Smoking Service in the United Kingdom. ${ }^{55}$ Even though most people understand the advantages of a healthier behavior, many are unable to make a change in their lifestyle. Moving from contemplation to action is influenced by complex personal, cultural, and socioeconomic factors. In addition, changes may not be stable, and individuals may fall back to smoking. ${ }^{56}$ Nicotine replacement therapy represents the mainstay of strategies to quit smoking. ${ }^{57}$ Intensive individual behavior therapy interventions are considered as an alternative when nicotine replacement therapy fails or in situations where people are reluctant to use it.

As already mentioned, dermatologists could play a major role in influencing people to change their behavior. This may require training and motivations. A few surveys have suggested that dermatologists may lack sufficient knowledge and educational skills or may be reluctant to promote lifestyle interventions in their practice. ${ }^{58}$ In consideration of the importance of healthier behaviors for many skin diseases, including psoriasis, a curriculum should be developed to teach dermatologists on patient-centered communication and on strategies aimed at changing behavior. ${ }^{59}$

\section{Disclosure}

The Study Center of the Italian Group for Epidemiologic Research in Dermatology (GISED), which the author leads, has received unrestricted research grant from pharmaceutical and cosmetics companies (Ketoprofen Consortium, Menarini, Abbvie, and L'Oreal). The author has served as a consultant or has been a part of advisory boards for Abbvie, Pfizer, Amgen, Novartis, Menarini, Boehringer Ingelheim, and Sanofi. The author reports no other conflicts of interest in this work.

\section{References}

1. US Department of Health and Human Services. How Tobacco Smoke Causes Disease: The Biology and Behavioral Basis for SmokingAttributable Disease: A Report of the Surgeon General. Atlanta, GA: US Department of Health and Human Services, Centers for Disease Control and Prevention, National Center for Chronic Disease Prevention and Health Promotion, Office on Smoking and Health; 2010.

2. Brandt AM. The Cigarette Century: The Rise, Fall, and Deadly Persistence of the Product That Defined America. New York, NY: Basic Books; 2007. 
3. International Agency for Research on Cancer. IARC Monographs on the Evaluation of the Carcinogenic Risks to Humans: Tobacco Smoke and Involuntary Smoking. Vol 83. Lyon, France: International Agency for Research on Cancer; 2004.

4. Morris PB, Ference BA, Jahangir E, et al. Cardiovascular effects of exposure to cigarette smoke and electronic cigarettes: clinical perspectives from the prevention of cardiovascular disease section leadership council and early career councils of the American College of Cardiology. J Am Coll Cardiol. 2015;66(12):1378-1391.

5. Rennard SI, Drummond MB. Early chronic obstructive pulmonary disease: definition, assessment, and prevention. Lancet. 2015;385(9979): $1778-1788$.

6. Sopori M. Effects of cigarette smoke on the immune system. Nat Rev Immunol. 2002;2(5):372-377.

7. Rodgman A, Perfetti TA. The Chemical Components of Tobacco and Tobacco Smoke. Boca Raton, FL: CRC Press, Taylor \& Francis Group; 2009.

8. BenowitzNL. Nicotine addiction. NEnglJMed.2010;362(24):2295-2303.

9. Tanner JA, Chenoweth MJ, Tyndale RF. Pharmacogenetics of nicotine and associated smoking behaviors. Curr Top Behav Neurosci. 2015;23:37-86.

10. Fowles J, Dybing E. Application of toxicological risk assessment principles to the chemical constituents of cigarette smoke. Tob Control. 2003;12(4):424-430.

11. Bekki K, Uchiyama S, Ohta K, Inaba Y, Nakagome H, Kunugita N. Carbonyl compounds generated from electronic cigarettes. Int J Environ Res Public Health. 2014;11(11):11192-11200.

12. Mucha L, Stephenson J, Morandi N, Dirani R. Meta-analysis of disease risk associated with smoking, by gender and intensity of smoking. Gend Med. 2006;3(4):279-291.

13. Benowitz NL, Hall SM, Stewart S, Wilson M, Dempsey D, Jacob P 3 rd. Nicotine and carcinogen exposure with smoking of progressively reduced nicotine content cigarette. Cancer Epidemiol Biomarkers Prev. 2007;16(11):2479-2485.

14. Shopland DR. Historical perspective: the low tar lie. Tob Control. 2001;10(Suppl 1):i1-i3.

15. Benowitz NL. Smokeless tobacco as a nicotine delivery device: harm or harm reduction? Clin Pharmacol Ther. 2011;90(4):491-493.

16. Farsalinos KE, Le Houezec J. Regulation in the face of uncertainty: the evidence on electronic nicotine delivery systems (e-cigarettes). Risk Manag Healthc Policy. 2015;8:157-167.

17. Jensen RP, Luo W, Pankow JF, Strongin RM, Peyton DH. Hidden formaldehyde in e-cigarette aerosols. N Engl J Med. 2015;372(4):392-394.

18. Naldi L, Mercuri SR. Smoking and psoriasis: from epidemiology to pathomechanisms. J Invest Dermatol. 2009;129(12):2741-2743.

19. Gilbert DG, Gilbert BO. Personality, psychopathology, and nicotine response as mediators of the genetics of smoking. Behav Genet. 1995;25(2):133-147.

20. Caporaso N, Gu F, Chatterjee N, et al. Genomewide and candidate gene association study of cigarette smoking behaviors. PLoS One. 2009;4:e4653.

21. Lutz SM, Hokanson JE. Genetic influences on smoking and clinical disease. Understanding behavioral and biological pathways with mediation analysis. Ann Am Thorac Soc. 2014;11(7):1082-1083.

22. Li MD, Cheng R, Ma JZ, Swan GE. A meta-analysis of estimated genetic and environmental effects on smoking behavior in male and female adult twins. Addiction. 2003;98(1):23-31.

23. Loukola A, Hallfors J, Korhonen T, Kaprio J. Genetics and smoking. Curr Addict Rep. 2014;1(1):75-82.

24. Armstrong AW, Harskamp CT, Dhillon JS, Armstrong EJ. Psoriasis and smoking: a systematic review and meta-analysis. Br J Dermatol. 2014;170(2):304-314.

25. Naldi L, Chatenoud L, Linder D, et al. Cigarette smoking, body mass index, and stressful life events as risk factors for psoriasis: results from an Italian case-control study. J Invest Dermatol. 2005;125(1):61-67.

26. La Vecchia C, Gallus S, Naldi L. Tobacco and skin disease. Dermatology. 2005;211(2):81-83.
27. Jin Y, Yang S, Zhang F, et al. Combined effects of HLA-Cw6 and cigarette smoking in psoriasis vulgaris: a hospital-based case-control study in China. J Eur Acad Dermatol Venereol. 2009,23(2):132-137.

28. Yin XY, Cheng H, Wang WJ, et al. TNIP1/ ANXA6 and CSMD1 variants interacting with cigarette smoking, alcohol intake affect risk of psoriasis. J Dermatol Sci. 2013;70(2):94-98.

29. Quan C, Zhu KJ, Zhang C, et al. Combined effects of the BDNF rs6265 (Val66Met) polymorphism and environment risk factors on psoriasis vulgaris. Mol Biol Rep. 2014;41(10):7015-7022.

30. Krämer U, Esser C. Cigarette smoking, metabolic gene polymorphism, and psoriasis. J Invest Dermatol. 2006;126(3):693-694.

31. Armstrong AW, Armstrong EJ, Fuller EN, Sockolov ME, Voyles SV. Smoking and pathogenesis of psoriasis: a review of oxidative, inflammatory and genetic mechanisms. Br J Dermatol. 2011;165(6):1162-1168.

32. Yanagita M, Kobayashi R, Kojima Y, Mori K, Murakami S. Nicotine modulates the immunological function of dendritic cells through peroxisome proliferator-activated receptor- $\gamma$ upregulation. Cell Immunol. 2012;274(1-2):26-33.

33. Jeong SH, Park JH, Kim JN, et al. Up-regulation of TNF- $\alpha$ secretion by cigarette smoke is mediated by Egr-1 in HaCaT human keratinocytes. Exp Dermatol. 2010;19(8):e206-e212.

34. Csordas A, Bernhard D. The biology behind the atherothrombotic effects of cigarette smoke. Nat Rev Cardiol. 2013;10(4)219-230.

35. Fortes C, Mastroeni S, Leffondré K, et al. Relationship between smoking and the clinical severity of psoriasis. Arch Dermatol. 2005;141(12): $1580-1584$

36. Gupta MA, Gupta AK, Watteel GN. Cigarette smoking in men may be a risk factor for increased psoriasis of the extremities. $\mathrm{Br} J$ Dermatol. 1996;135(5):859-860.

37. Raychaudhuri SP, Gross J. Psoriasis risk factors: role of lifestyle practices. Cutis. 2000;66(5):348-352.

38. Kinahan CE, Mazloom S, Fernandez AP. Impact of smoking on response to systemic treatment in patients with psoriasis: a retrospective casecontrol study. Br J Dermatol. 2015;172(2):428-436.

39. Thorneloe RJ, Bundy C, Griffiths CE, Ashcroft DM, Cordingley L. Adherence to medication in patients with psoriasis: a systematic literature review. Br J Dermatol. 2013;168(1):20-31.

40. Di Lernia V, Ricci C, Lallas A, Ficarelli E. Clinical predictors of nonresponse to any tumor necrosis factor (TNF) blockers: a retrospective study. J Dermatolog Treat. 2014;25(1):73-74.

41. Zhu KJ, Quan C, Zhang C, et al. Combined effect between CHRNB3CHRNA6 region gene variant (rs6474412) and smoking in psoriasis vulgaris severity. Gene. 2014;544(2):123-127.

42. Naldi L. Epidemiology of psoriasis. Curr Drug Targets Inflamm Allergy. 2004;3(2):121-128.

43. Naldi L, Chatenoud L. Psoriasis et tumeurs : un lien plus qu'un hasard. [Psoriasis and cancer: more than a chance link]. Ann Dermatol Venereol. 2006;133(3):221-223. French.

44. Rakkhit T, Wong B, Nelson TS, Hansen CB, Papenfuss JS, Panko J. Time to development of psoriatic arthritis decreases with smoking prior to psoriasis onset and increases with smoking after psoriasis onset. $J$ Invest Dermatol. 2007;127(Suppl 2):52.

45. Tey HL, Ee HL, Tan AS, et al. Risk factors associated with having psoriatic arthritis in patients with cutaneous psoriasis. J Dermatol. 2010;37(5):426-430.

46. Li W, Han J, Qureshi AA. Smoking and risk of incident psoriatic arthritis in US women. Ann Rheum Dis. 2012;71(6):804-808.

47. Tillett W, Jadon D, Shaddick G, et al. Smoking and delay to diagnosis are associated with poorer functional outcome in psoriatic arthritis. Ann Rheum Dis. 2013;72(8):1358-1361.

48. Højgaard P, Glintborg B, Hetland ML, et al. Association between tobacco smoking and response to tumour necrosis factor $\alpha$ inhibitor treatment in psoriatic arthritis: results from the DANBIO registry. Ann Rheum Dis. 2015;74(12):2130-2136.

49. Skudutyte-Rysstad R, Slevolden EM, Hansen BF, Sandvik L Preus HR. Association between moderate to severe psoriasis and periodontitis in a Scandinavian population. BMC Oral Health. 2014;14:139. 
50. Antal M, Braunitzer G, Mattheos N, Gyulai R, Nagy K. Smoking as a permissive factor of periodontal disease in psoriasis. PLoS One. 2014;14:139.

51. Kalb RE, Fiorentino DF, Lebwohl MG, et al. Risk of serious infection with biologic and systemic treatment of psoriasis: results from the psoriasis longitudinal assessment and registry (PSOLAR). JAMA Dermatol. 2015;151(9):961-969.

52. Pugliese D, Guidi L, Ferraro PM, et al. Paradoxical psoriasis in a large cohort of patients with inflammatory bowel disease receiving treatment with anti-TNF $\alpha$ : 5-year follow-up study. Aliment Pharmacol Ther. 2015;42(7):880-888.

53. Peto R, Lopez AD, Boreham J, Thun M, Heath C Jr. Mortality from smoking in developed countries: indirect estimates from national vital statistics. Lancet. 1992;339(8804):1268-1278.

54. McIvor A, Kayser J, Assaad JM, et al. Best practices for smoking cessation interventions in primary care. Can Respir J. 2009;16(4):129-134
55. Dobbie F, Hiscock R, Leonardi-Bee J, et al. Evaluating long-term outcomes of NHS stop smoking services (ELONS): a prospective cohort study. Health Technol Assess. 2015;19(95):1-156.

56. Norcross JC, Krebs PM, Prochaska JO. Stages of change. J Clin Psychol. 2011;67(2):143-154.

57. Fiore MC, Baker TB. Clinical practice. Treating smokers in the health care setting. N Engl J Med. 2011;365(13):1222-1231.

58. Nelson PA, Keyworth C, Chisholm A, et al. Identification and Management of Psoriasis-Associated Co-morbidiTy (IMPACT) Team. "In someone's clinic but not in mine" - clinicians' views of supporting lifestyle behaviour change in patients with psoriasis: a qualitative interview study. Br J Dermatol. 2014;171(5):1116-1122.

59. Naldi L. Lifestyle intervention should be an essential component of medical care for skin disease: a challenging task. Br J Dermatol. 2014;171(5) 934-935.
Psoriasis: Targets and Therapy

\section{Publish your work in this journal}

Psoriasis: Targets and Therapy is international, peer-reviewed, open access journal focusing on psoriasis, nail psoriasis, psoriatic arthritis and related conditions, identification of therapeutic targets and the optima use of integrated treatment interventions to achieve improved outcomes

\section{Dovepress}

and quality of life. Visit http://www.dovepress.com/testimonials.php to read real quotes from published authors. 